\title{
Fatores de risco associados à leptospirose em cães do município de Londrina-PR
}

\section{Risk factors associated to leptospirosis in dogs in Londrina City - PR}

\author{
Adriana Martins Vieira Querinoํㅜㄹ Ádina Cléia Botazzo Delbem¹; Rosângela Claret de \\ Oliveira $^{1}$; Francielle Gibson da Silva ${ }^{1}$; Ernst Eckehardt Müller ${ }^{2}$; \\ Roberta Lemos Freire ${ }^{2}$; Julio Cesar de Freitas*2
}

\begin{abstract}
Resumo
A leptospirose é uma zoonose reemergente associada ao convívio próximo entre o cão e o homem. É importante determinar se os fatores de risco da leptospirose canina são semelhantes aos do homem, ou são variáveis exclusivas ao hábito e manejo da espécie. Os objetivos deste trabalho foram identificar variáveis individuais e ambientais, associadas a maior freqüência de cães soropositivos para leptospira, atendidos no Hospital Veterinário da Universidade Estadual de Londrina. Foram estudados 160 cães de ambos os sexos e não vacinados contra leptospirose, entre março de 1997 e abril de 1998. Todos os animais foram submetidos à prova de aglutinação microscópica e ao exame direto da urina. Para cada um deles foi utilizado um questionário epidemiológico que procurou investigar variáveis que poderiam estar associadas a essa infecção. Os resultados obtidos foram submetidos ao teste $\chi^{2}$. Foram detectados títulos de anticorpos $\geq 100$ em 40 cães, sendo em maior freqüência contra o sorovar pyrogenes $(45,00 \%)$ e 24 animais foram positivos no exame direto da urina. A análise das variáveis apontou como fator de risco para a leptospirose canina,o hábito de caçar roedor $(\mathrm{OR}=4,22 ; 1,89 \leq \mathrm{IC} 95 \% \leq 9,50)$, a presença de áreas alagadiças próximas às residências $(\mathrm{OR}=2,86 ; 1,32 \leq \mathrm{IC} 95 \% \leq 6,22)$ e o acesso à rua $(\mathrm{OR}=2,57$; $1,19 \leq \mathrm{IC} 95 \% \leq 5,59)$. Os resultados servem como alerta em relação a possibilidade de exposição humana a alguns fatores de risco para a leptospirose a que estão expostos estes cães.
\end{abstract}

Palavras-chave: Leptospirose, cão, fator de risco, diagnóstico, sorologia.

\begin{abstract}
Leptosporosis is a re-emergent zoonosis associated to close contact between man and dogs. It is important to determine whether the canine leptospirosis risk factors are similar to those of man or are variables exclusive to the species habitat and management. The objective of this study was to identify the individual and environmental variables most frequently associated with seropositive dogs attended in the Veterinary Hospital at Londrina State University. One hundred and sixty dogs of both sexes not vaccinated against leptospirosis were studied over a 13-month period (march 1997 to april 1998). All the animals were submitted to the microscopic aglutination test and to the direct urine test. An epidemiological questionnaire filled out for each one to investigate variables that could be associated with this infection
\end{abstract}

\footnotetext{
1 Aluna de pós- graduação do Departamento de Medicina Veterinária Preventiva; Centro de Ciências Agrárias; Universidade Estadual de Londrina; Londrina - Paraná - Brasil.

2 Professor do Departamento de Medicina Veterinária Preventiva; Centro de Ciências Agrárias; Universidade Estadual de Londrina; Londrina - Paraná - Brasil.

" Autor para correspondência: Prof. Dr. Julio Cesar de Freitas - E-mail: freitasj@uel.br - Tel. (43) 3371-4765; Fax: (43) 33714714; Rodovia Celso Garcia Cid (PR 445), km 380; CEP: 86051-990; Caixa Postal:6001.
} 
and the results obtained were submitted to the $\chi^{2}$ test. Antibody titers $\geq 100$ were detected in 40 dogs and were more frequent against the pyrogenes serovar $(45,00 \%)$. Twenty-four animals were positive in the direct urine test. The analysis of the variables pointed as a risk factor for canine leptospirosis the habit of hunting rodent $(\mathrm{OR}=4.22,1.89 \leq \mathrm{IC} 95 \% \leq 9.50)$, the presence of flooded areas near the houses $(\mathrm{OR}=$ $2.86,1.32 \leq \mathrm{IC} 95 \% \leq 6.22)$ and access to the street $(\mathrm{OR}=2.57,1.19 \leq \mathrm{IC} 95 \% \leq 5.59)$. The results serve as a warning about the possibility of human exposure to some risk factors that these dogs are exposed to.

Key words: Leptospirosis, dog, risk factor, diagnostic, serology.

\section{Introdução}

A leptospirose é uma das principais zoonoses com distribuição mundial, tendo sido descrita em todos os tipos de vertebrados de sangue quente. Os cães desempenham um papel importante na epidemiologia da leptospirose humana, pela proximidade aos seres humanos (WEEKES; EVERARD; LEVETT, 1997), por serem reservatórios do sorovar canicola (BOLIN, 1996) e pela sua capacidade de eliminar leptospiras vivas através da urina durante vários meses (AVILA et al., 1998).

A ocorrência dos sorovares de Leptospira spp varia de acordo com a região geográfica e em função dos aspectos ecológicos que caracterizam cada uma destas regiões (BOLIN, 1996). Além disso, a prevalência desta enfermidade em cães é influenciada por vários fatores, dentre eles os índices pluviométricos e a presença de roedores (FAINE, 1999).

O crescimento urbano desordenado ocasiona problemas de saneamento básico e a conseqüente proliferação de roedores, que são os principais portadores de leptospiras no meio urbano, principalmente do sorovar icterohaemorrhagiae (YASUDA; SANTA ROSA; YANAGUITA, 1980). Estudos realizados na cidade de Detroit, nos EUA, mostraram que $75,00 \%$ dos ratos (Rattus norvegicus) de esgoto eram portadores crônicos de leptospiras (THIERMANN, 1977). No Brasil, estudos também feitos com ratos em São Paulo-SP (FORATTINI, 1947), em Salvador-BA (ANDRADE; OLIVEIRA, 1954) e em Duque de Caxias-RJ
(LILENBAUM et al., 1993), indicaram índices de infecção de $31,40 \%, 30,00 \%$ e 36,20\%, respectivamente.

Uma vez estabelecida a proximidade entre a espécie canina e a humana, pouco se estudou para verificar se os cães estão sob a ação de fatores de risco iguais ou próximos aos nossos, ou ainda variáveis exclusivas ao hábito e manejo da espécie. Rubel et al. (1997) realizaram um estudo transversal analisando variáveis ambientais e individuais associadas ao risco para a leptospirose canina onde concluíram que a presença de água estagnada próxima à residência aumentou o risco da infecção. Resultado semelhante foi encontrado por Douglin et al. (1997) que observaram que pessoas que caminhavam em áreas com água estagnada ou poças têm 25,62 vezes mais chance de ser reagente para a leptospirose. Este fator de risco determinado para o cão e para o homem é um exemplo da simultaneidade de variáveis ambientais que podem oferecer risco para ambos, visto o compartilhamento do ambiente e de seu asseio.

Pelo convívio bastante próximo entre o cão e o homem, é importante determinar se os fatores de risco da leptospirose canina são iguais ou próximos aos do homem, ou são variáveis exclusivas ao hábito e manejo da espécie.

Os objetivos deste trabalho foram identificar variáveis individuais e ambientais, associadas a maior freqüência de cães soropositivos para leptospira atendidos no Hospital Veterinário da Universidade Estadual de Londrina (HV-UEL). 


\section{Material e Métodos}

\section{Animais}

Entre março de 1997 e abril de 1998 foram estudados 160 cães, sendo 140 de área urbana e 20 de área rural, de ambos os sexos, com suspeita clínica de leptospirose (FAINE, 1999) porém não vacinados contra este microrganismo, atendidos no HV-UEL.

\section{Soro e urina}

No exame clínico, foi colhida uma amostra de sangue e uma de urina (obtida por cistocentese ou por sonda estéril) de cada animal estudado, que foram enviadas imediatamente ao Laboratório de Leptospirose do Departamento de Medicina Veterinária Preventiva da UEL. As amostras de urina foram examinadas no máximo 30 minutos após a colheita e as de sangue, mantidas em temperatura ambiente até que houvesse a retração do coágulo. $\mathrm{O}$ soro obtido foi mantido a $-20^{\circ} \mathrm{C}$ até o momento de realização dos exames.

\section{Exames realizados}

Das amostras de urina, foram utilizados $10 \mu \mathrm{l}$ para o exame direto em microscopia de campo escuro (FAINE, 1999). Foram consideradas positivas as amostras que apresentaram estruturas com morfologia e movimentação compatíveis às de espiroquetas.

Os soros foram submetidos à prova de aglutinação microscópica com antígenos vivos (RYU, 1970), utilizando 21 sorovares de Leptospira interrogans. Os antígenos foram mantidos a $28^{\circ} \mathrm{C}$ por 5 a 10 dias em meio de Elinghausen-McCullough-JohnsonHarris (EMJH) ${ }^{1}$ modificado com albumina bovina. Todos os soros que apresentaram $50 \%$ ou mais de leptospiras aglutinadas na diluição 1:100 foram considerados positivos e então diluídos seriadamente para a determinação da diluição máxima positiva.

\footnotetext{
${ }^{1}$ Leptospira Medium Base EMJH-DIFCO Laboratories
}

\section{Variáveis analisadas}

Para a determinação das variáveis associadas a leptospirose no grupo estudado, foi utilizado um questionário epidemiológico para cada animal. Analisaram-se as seguintes variáveis: cão de origem urbana ou rural, existência de áreas alagadiças próximas às residências, contato com outros animais, acesso à rua, hábito de caçar roedor, condições de saneamento básico (coleta de lixo, origem da água , destino dado ao esgoto) e presença de terrenos baldios nos arredores das residências.

\section{Análise estatística}

Os resultados obtidos após o estudo das variáveis foram submetidos à análise estatística através do Teste do Qui-Quadrado $\left(\chi^{2}\right)$ corrigido de Yates, Teste de Fisher e do Cálculo de Odds Ratio (OR), mediante o programa estatístico Epi Info versão 6.04 (DEAN; DEAN; COULOMBIER, 1994). Adotou-se 95\% de intervalo de confiança.

\section{Resultados}

Dos 160 cães pesquisados, $49(30,52 \%)$ apresentaram resultados considerados positivos para leptospirose, sendo $25(51,02 \%)$ positivos apenas na aglutinação microscópica, nove $(18,37 \%)$ somente no exame direto da urina em microscopia de campo escuro e $15(30,61 \%)$ tanto no exame direto da urina quanto na sorologia.

Considerando-se somente os maiores títulos positivos encontrados na prova de aglutinação microscópica, sete $(17,50 \%)$ cães apresentaram títulos de 100, sete $(17,50 \%)$ de 200, $12(30,00 \%)$ de 400 , cinco $(12,50 \%)$ de 800 , três $(7,50 \%)$ de 1.600 , dois $(5,00 \%)$ de 3.200 , três $(7,50 \%)$ de 6.400 e um $(2,50 \%)$ de 12.800. Em 19 (47,50\%) cães foram detectados anticorpos apenas contra um sorovar de Leptospira interrogans e em $21(52,50 \%)$ cães contra dois ou mais sorovares simultaneamente. Os sorovares mais freqüentes foram pyrogenes e icterohaemorrhagiae (Tabela 1). 
Tabela 1. Resultados positivos na prova de aglutinação microscópica, com 21 sorovares, em 40 amostras de soro de cães atendidos no Hospital Veterinário da Universidade Estadual de Londrina, entre março de 1997 e abril de 1998.

\begin{tabular}{lcc}
\hline \multicolumn{1}{c}{ Sorovar } & n & \% \\
\hline pyrogenes & 18 & $45,00 \%$ \\
icterohaemorrhagiae & 16 & $40,00 \%$ \\
copenhageni & 9 & $22,50 \%$ \\
bataviae & 9 & $22,50 \%$ \\
bratislava & 7 & $17,50 \%$ \\
autumnalis & 6 & $15,00 \%$ \\
grippotyphosa & 6 & $15,00 \%$ \\
canicola & 3 & $7,50 \%$ \\
fort bragg & 3 & $7,50 \%$ \\
butembo & 3 & $7,50 \%$ \\
pomona & 2 & $5,00 \%$ \\
hardjo & 1 & $2,50 \%$ \\
wolffi & 1 & $2,50 \%$ \\
\hline
\end{tabular}

$\mathrm{n}=$ número de amostras positivas

$\%=$ percentagem de amostras positivas
A Tabela 2 mostra os resultados de OR obtidos pela análise das variáveis. Três variáveis foram consideradas fatores de risco para os cães: acesso à rua $(\mathrm{p}=0,012$ e $\mathrm{OR}=2,65)$; hábito de caçar roedor $(\mathrm{p}=0,0002$ e $\mathrm{OR}=4,22)$ e existência de áreas alagadiças próximas às residências $(\mathrm{p}=0,006 \mathrm{e} \mathrm{OR}$ $=2,86$ ). Não houve diferença significativa em relação às variáveis: cães de zona urbana $(p=0,4757 \mathrm{e} O R=$ $0,62)$; cães que tinham contato com outros animais $(\mathrm{p}=0,6524$ e $\mathrm{OR}=1,26)$; residência sem coleta de lixo $(\mathrm{p}=0,97$ e $0 \mathrm{R}=1,79)$ e água tratada $(\mathrm{p}=0,76 \mathrm{e}$ $\mathrm{OR}=1,33)$; cães provenientes de residências com terrenos baldios próximos ( $\mathrm{p}=0,143$ e $\mathrm{OR}=1,88)$ e sem esgoto da rede pública $(\mathrm{p}=0,130$ e $\mathrm{OR}=1,82)$.

Tabela 2. Resultado das variáveis analisadas para a determinação dos fatores de risco para leptospirose em 160 cães atendidos no Hospital Veterinário da Universidade Estadual de Londrina entre março de 1997 e abril de 1998.

\begin{tabular}{|c|c|c|c|c|c|}
\hline VARIÁVEIS & $\begin{array}{c}\text { Cães } \\
\text { soropositivos }\end{array}$ & $\begin{array}{c}\text { Cães } \\
\text { soronegativos }\end{array}$ & $\chi^{2}$ & $\mathrm{p}$ & OR (IC) \\
\hline Cães de origem urbana & $41 / 49$ & $99 / 111$ & 0,51 & 0,475 & $0,62(0,22 \leq \mathrm{IC} \leq 1,81)$ \\
\hline $\begin{array}{l}\text { Contato com outros } \\
\text { animais }\end{array}$ & $33 / 49$ & $69 / 111$ & 0,20 & 0,652 & $1,26(0,58 \leq \mathrm{IC} \leq 2,72)$ \\
\hline Acesso à rua & $34 / 49$ & $52 / 111$ & 6,07 & 0,0013 & $2,57 * *(1,19 \leq \mathrm{IC} \leq 5,59)$ \\
\hline Hábito de caçar roedor & $27 / 44$ & $29 / 106$ & 13,95 & 0,000 & $4,22 * *(1,89 \leq \mathrm{IC} \leq 9,50)$ \\
\hline $\begin{array}{l}\text { Residência próxima a } \\
\text { terrenos baldios }\end{array}$ & $37 / 49$ & $69 / 111$ & 2,14 & 0,143 & $1,88(0,83 \leq \mathrm{IC} \leq 4,29)$ \\
\hline $\begin{array}{l}\text { Residência com água } \\
\text { tratada }\end{array}$ & $48 / 49$ & $108 / 111$ & $0,64 *$ & 0,641 & $1,33(0,12 \leq \mathrm{IC} \leq 34,15)$ \\
\hline $\begin{array}{l}\text { Residência sem esgoto de } \\
\text { rede pública }\end{array}$ & $32 / 49$ & $56 / 110$ & 2,29 & 0,130 & $1,82(0,86 \leq \mathrm{IC} \leq 3,87)$ \\
\hline $\begin{array}{l}\text { Residência sem coleta de } \\
\text { lixo }\end{array}$ & $48 / 49$ & $107 / 111$ & $0,52 *$ & 0,513 & $1,79(0,18 \leq \mathrm{IC} \leq 43,30)$ \\
\hline $\begin{array}{l}\text { Áreas alagadiças próximas } \\
\text { às residências }\end{array}$ & $23 / 48$ & $27 / 111$ & 7,59 & 0,005 & $2,86 * *(1,32 \leq \mathrm{IC} \leq 6,22)$ \\
\hline
\end{tabular}

\section{$\mathrm{P} \leq 0,05$}

*Teste de Fisher

** Fator de risco 


\section{Discussão}

Embora as infecções causadas pelos sorovares canicola e icterohaemorrhagiae sejam tradicionalmente associadas à leptospirose em cães, não só no Brasil (SANTA ROSA et al., 1969-70; YASUDA; SANTA ROSA; YANAGUITA, 1980; AVILA et al., 1998) como em outros países (THIERMANN, 1980; VENKATARAMAN; NEDUNCHELLIYAN, 1991), no presente estudo, anticorpos contra o sorovar canicola foram encontrados em somente $7,50 \%$ dos cães positivos. Entretanto, detectaram-se anticorpos contra o sorovar icterohaemorrhagiae em 40,00\% dos cães positivos. Esta alteração do perfil sorológico clássico também foi verificada por Scanziani et al. (1994). As mudanças observadas nos sorovares mais encontrados nos cães dos Estados Unidos e Canadá foram atribuídas tanto às vacinações contra os sorovares canicola e icterohaemorrhagiae, quanto a um maior contato entre cães e animais silvestres, o que determinou infecções por outros sorovares não presentes nas vacinas comerciais (BOLIN, 1996).

Neste trabalho, anticorpos contra o sorovar pyrogenes foram detectados em $45,00 \%$ dos cães positivos. Além da importância deste sorovar para os cães, ele é um dos mais patogênicos para o homem, com repercussão na saúde pública (STANCHI; ARIAS, 1997). O sorovar pyrogenes foi isolado do rato d'água (Nectomys squamipes) no Brasil (SANTA ROSA et al., 1980) e de cães na Argentina (SZYFRES, 1976). Estudos sorológicos feitos com cães no Brasil (CARVALHO; TORRES; RIBEIRO, 1979), Argentina (RUBEL et al., 1997) e Filipinas (TOPACIO, 1974) revelaram frequiências altas deste sorovar.

Vários autores estudaram fatores de risco associados à leptospirose em diferentes espécies animais (PEREIRA; ANDRADE, 1990; RUBEL et al., 1997; MURHEKAR et al., 1998). Em cães de um bairro da Grande Buenos Aires, foi demonstrada soroprevalência maior entre aqueles que tinham o hábito de caça do que entre os não caçadores; contudo, não houve diferença significativa entre os dois grupos estudados (RUBEL et al., 1997). Os resultados obtidos no presente trabalho indicaram que o hábito de caçar roedor foi um dos principais fatores de risco. Os cães que possuíam este hábito tiveram um risco 4,22 vezes maior de ter a infecção em relação aos não caçadores. Este fator de risco pode explicar a alta ocorrência, nos cães estudados, de anticorpos contra os sorovares pyrogenes e icterohaemorrhagiae. Entretanto, não se pode descartar a possibilidade da infecção ter ocorrido neste grupo de cães, por outros meios que não o de caçar roedor.

A presença de áreas alagadiças próximas às residências onde eram mantidos os cães constituiu um fator de risco. Estes animais tiveram um risco 2,86 vezes maior de se infectarem do que os procedentes de locais não alagadiços. Isto pode ser explicado pelo fato da leptospira ter uma estreita associação com fatores ambientais. Uma estrutura ecológica favorável permite a manutenção das leptospiras no ambiente e oferece inúmeras possibilidades de transmissão do agente (BLENDEN, 1976). A água é considerada o fator ambiental mais importante na manutenção deste microrganismo (ANDRE-FONTAINE; GANIERE, 1990) e, uma vez contaminada com a urina de animais infectados, constitui um elemento bastante significativo na transmissão da leptospirose (SZYFRES, 1976; WEEKES; EVERARD; LEVETT, 1997). Os resultados do presente estudo estão de acordo com os obtidos na Grande Buenos Aires (RUBEL et al., 1997) e em São Paulo (YASUDA; SANTA ROSA; YANAGUITA, 1980), os quais demonstraram que a possível presença de ratos e cães compartilhando de áreas alagadiças constitui uma importante forma de contaminação ambiental. Douglin et al. (1997) também encontraram uma predisposição à ocorrência de leptospirose em humanos que caminhavam em áreas alagadiças aumentada em 25,62 vezes.

Os resultados deste trabalho mostraram que os cães com acesso à rua tiveram um risco 2,57 vezes maior de se infectarem em relação àqueles que não tiveram acesso à rua. No estudo epidemiológico feito 
em Buenos Aires, o acesso à rua também foi um fator de risco significativo (RUBEL et al., 1997). Deve-se considerar que este hábito pode propiciar inúmeras possibilidades de infecção pelo contato direto ou indireto com outros animais ou através do acesso à áreas alagadiças.

É provável, que o hábito de caçar roedor, o acesso à rua, a presença de áreas alagadiças, sejam fatores que estejam interligados, favorecendo a disseminação da leptospirose entre os cães.

Os resultados obtidos servem como um alerta em relação ao potencial zoonótico da leptospirose canina (RUBEL et al., 1997; WEEKES; EVERARD; LEVETT, 1997; AVILA et al., 1998) e também no que diz respeito à exposição dos proprietários e comunicantes dos cães a alguns dos mesmos fatores de risco a que estiveram expostos os cães.

\section{Referências}

ANDRADE, Z.; OLIVEIRA, J. C. Estudo sobre a leptospirose na Bahia. Boletim da Fundação Gonçalo Muniz, Canela, v.3, p.1-28, 1954.

ANDRE-FONTAINE, G.; GANIERE, J. P. New topics on leptospirosis. Comparative Immunology, Microbiology And Infectious Diseases, Oxford, v.13, n.3, p.163-168, 1990.

AVILA, M. O.; FURTADO, L. R. I. ; TEIXEIRA, M. M.; ROSADO, R.. L. I.; MARTINS, L. F. S. ; BROD, C. S. Aglutininas anti-leptospíricas em cães na área de influência do Centro de Controle de Zoonoses, Pelotas, RS, Brasil, 1995. Ciência Rural, Santa Maria, v.28, n.1, p.107-110, 1998.

BLENDEN, D. C. Aspectos epidemiologicos de la leptospirosis. In: REUNION INTERAMERICANA SOBRE EL CONTROL DE LA FIEBRE AFTOSA Y OTRAS ZOONOSES, 8., 1976, Washington. Anais... Washington: Organizacion Panamericana de La Salud, 1976. p.160-168.

BOLIN, C. A. Diagnosis of leptospirosis: a reemerging disease of companion animals. Seminars in Veterinary Medicine and Surgery (Small Animal), Philadelphia, v.11, n.3, p.166-171, 1996.

CARVALHO, A. G.; TORRES, A. J.; RIBEIRO, J. L. Pesquisa de aglutininas anti-leptospira em cães de Goiânia. Revista de Patologia Tropical, Goiânia, v.8, n.1-2, p.5-8, 1979.
DEAN, A. G.; DEAN, J. A.; COULOMBIER, D. Epi Info version 6.04. A word processing, database and statistics program for epidemiology on microcomputers. Atlanta: Centers for Disease Control and Prevention, 1994.

DOUGLIN, C. P.; JORDAN, C.; ROCK, R.; HURLEY, A.; LEVETT, P. N. Risk factors for severe leptospirosis in the Parish of St. Andrew, Barbados. Emergence Infectious Disease, v.3, n.1, jan-mar. 1997. Disponível em: <http:// www.cdc.gov/ncidod/eid/vol3no1/douglin.htm>. Acesso em: 27 ago. 2000.

FAINE, S. Leptospira and Leptospirosis. $2^{\text {th }}$ ed. Melbourne: MediSci, 1999.

FORATTINI, O. P. Presença de leptospiras nos ratos da cidade de São Paulo. Anais da Faculdade de Medicina da Universidade de São Paulo, São Paulo, v.23, p.5-27, 1947.

LILENBAUM, W.; RIBEIRO, V.; MARTIN, E.; BISPO, V. Estudo sorológico para a detecção de anticorpos antileptospira em Rattus norvegicus de Duque de Caxias, Rio de Janeiro, Brasil. Revista Latino-Americana de Microbiologia, Mexico, v.35, p.357-360, 1993.

MURHEKAR, M. V.; SUGUNAN, A.P.; VIJAYACHARI, P.; SHARMA, S.; SEHGAL, S.C. Risk factors in the transmission of leptospiral infection. Indian Journal Medical Research, New Delhi, v.107, p.218-223, 1998.

PEREIRA, M. M.; ANDRADE, J. Human leptospirosis in a slum area in the city of Rio de Janeiro, Brasil: a serological and epidemiological study. Memórias do Instituto Osvaldo Cruz, Rio de Janeiro, v.85, n.1, p.47-52, 1990.

RUBEL, D.; SEIJO, A; CERNIGOI, B.; VIALE, A.; WISNIVESKY, C. C. Leptospira interrogans en una población canina del Gran Buenos Aires: variables asociadas con la positividad. Revista Panamericana de Salud Pública, Washington, v.2, n.2, p.102-105, 1997.

RYU, E. Rapid microscopic agglutination test for leptospira without non-specific reaction. Bulletin Official of International Epizooties, Paris, v.73, n.1-2, p.49-58, 1970.

SANTA ROSA, C. A.; PESTANA DE CASTRO, A. F.; SILVA, A.; TERUYA, J. M. Nove anos de leptospirose no Instituto Biológico de São Paulo. Revista Instituto Adolfo Lutz, São Paulo, v.29-30, p.19-27, 1969-70.

SANTA ROSA, C. A.; SULZER, C. R.; YANAGUITA, R. M.; DASILVA, A. S. Leptospirosis in wildlife in Brazil: isolation of serovars canicola pyrogenes and grippotyphosa. International Journal of Zoonoses, Taipei, v.7, p.40-43, 1980.

SCANZIANI,E.; CALCATERRA, S.; TAGLIABUE, S.;LUINI, M.; GIUSTI, A. M.; TOMBA, M. Serological findings in cases of acute leptospirosis in the dog. Journal of Small Animal Practice, Gloucestershire, v.35, p.257-260, 1994. 
STANCHI, N. O.; ARIAS, D. O. Consideraciones de la leptospirosis. Avances en Medicina Veterinaria, Buenos Aires, v.1, p.20-21, 1997.

SZYFRES, S. Leptospirosis en América Latina y el Caribe. In REUNION INTERAMERICANA SOBREEL CONTROL DE LA FIEBRE AFTOSA Y OTRAS ZOONOSES, 8., 1976, Washington. Anais... Washington: Organizacion Panamericana de la Salud, 1976. p.124-141.

THIERMANN, A. B. Incidence of leptospirosis in the Detroit rat population. American Journal of Tropical Medicine and Hygiene, Northbrook, v.26, p.970-974, 1977.

THIERMANN, A. B. Canine leptospirosis in Detroit. American Journal of Veterinary Research, Schaumburg, v.41, n.10.p.1659-1661, 1980.
TOPACIO, T. M. Leptospirosis in animals and in the man in the Phillipines. VIII Serological incidence in native dogs. International Journal of Zoonoses, Taipei, v.1, p.2-42, 1974.

VENKATARAMAN, K. S.; NEDUNCHELLIYAN, S. Incidence of leptospiral jaundice among dogs in Madras. Indian Journal of Animal Health, Calcutta, p.53-54, 1991.

WEEKES, C. C.; EVERARD, C. O. R.; LEVETT, P. N. Seroepidemiology of canine leptospirosis on the island of Barbados. Veterinary Microbiology, Amsterdam, v.51, p.215-212, 1997.

YASUDA, P. H.; SANTA ROSA, C. A.; YANAGUITA, R. M. Variação sazonal na prevalência de leptospirose em cães de rua da cidade de São Paulo, Brasil. Revista Saúde Pública, São Paulo, v.14, p.589-596, 1980. 
